\title{
TRATAMIENTO MÍNIMAMENTE INVASIVO PARA RESTAURAR LA GUÍA ANTERIOR Y ESTÉTICA EN PACIENTE CON ERUP- CIÓN PASIVA ALTERADA
}

\author{
Minimally invasive treatment to restore anterior guidance and aesthetic in a pa- \\ tient with altered passive eruption
}

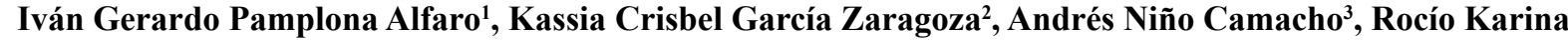 Cárdenas Delgado ${ }^{4}$, Andréa Dolores Correia Miranda Valdivia ${ }^{5 *}$}

\author{
${ }^{1}$ Cirujano Dentista, Residente en Posgrado Prostodoncia, Universidad Autónoma de Guadalajara. Guadalaja- \\ ra, Jalisco, México ivanpamplonaa@hotmail.com \\ ${ }^{2}$ Cirujano Dentista, Especialista en Periodoncia, Universidad Autónoma de Guadalajara. Guadalajara, Jalis- \\ co, México kassia.garcia@edu.uag.mx \\ ${ }^{3}$ Cirujano Dentista, Especialista en Rehabilitación Oral, Profesor en Posgrado Prostodoncia, Universidad \\ Autónoma de Guadalajara. Guadalajara, Jalisco, México andres.camacho@edu.uag.mx \\ ${ }^{4}$ Cirujano Dentista, Especialista en Periodoncia, Profesor en Posgrado Periodoncia, Universidad Autónoma \\ de Guadalajara. Guadalajara, Jalisco, México rocio.cardenas@edu.uag.mx

\begin{abstract}
${ }^{5}$ Doctora en Clínica Odontológica Integrada, Profesora Investigadora en el Departamento de Odontología andrea.correia@edu.uag.mx
\end{abstract} \\ Especializada, Posgrado Prostodoncia, Universidad Autónoma de Guadalajara. Guadalajara, Jalisco, México
}

DOI: https://doi.org/10.31984/oactiva.v6i3.641

\begin{abstract}
Resumen
La técnica de resinas inyectadas es un tratamiento innovador y poco invasivo ideal para pacientes con erupción pasiva alterada (EPA), cuando la ortodoncia no es una opción. Este tratamiento implica un desgaste mínimo o se puede realizar sin desgaste de tejido dental. Los alargamientos de corona también están indicados en estos casos, para obtener mejores resultados. Este reporte de caso presenta una rehabilitación estética y funcional, utilizando la técnica de resinas inyectadas en el sector antero-superior, en una paciente femenina de 17 años de edad que se presenta a las clínicas odontológicas de la Universidad Autónoma de Guadalajara referida por el departamento de ortodoncia. La paciente presentaba EPA lo cual se corroboró con un estudio periapical completo digital y realizando un sondeo periodontal. La opción de tratamiento ideal en pacientes jóvenes con EPA busca alternativas rehabilitadoras mínimamente invasivas para solucionar problemas estéticos y funcionales, cuando los pacientes no presentan desoclusiones posteriores en movimientos laterales y protrusivos. Esta opción de tratamiento es rápida de realizar y con resultados predecibles para el paciente. La técnica de gingivectomía que complementa la técnica de resinas inyectadas es realizada con electrobisturí y debe ser utilizada en casos específicos, teniendo conocimientos de los principios de la técnica, las habilidades para llevarla a cabo y el manejo adecuado de los tejidos donde se va a aplicar.
\end{abstract}

Palabras clave: Coronas con Frente Estético, Alargamiento de Corona, Resinas Compuestas, Erupción Dental. 


\begin{abstract}
The injected resin technique is an innovative and minimally invasive treatment, ideal for patients with altered passive eruption (EPA) when orthodontics is not an option. This treatment involves minimal or no tooth tissue wear. Crown lengthening is also indicated in these cases for better results. This case report presents an esthetic and functional rehabilitation, using the injected resin technique in the anterior-superior sector, in a 17-year-old female patient who presented to the dental clinics of the Universidad Autónoma de Guadalajara referred by the orthodontic department. The patient presented EPA which was corroborated with a complete digital periapical study and periodontal probing. The ideal treatment choice in young patients with EPA seeks minimally invasive rehabilitative alternatives to solve aesthetic and functional problems when patients do not present posterior unocclusions in lateral and protrusive movements. This treatment option is quick to perform and with predictable results for the patient. The gingivectomy technique that complements the injected resin technique is performed with an electrosurgical unit and must be used in specific cases, knowing the principles of the technique, the skills to perform it, and the proper handling of the tissues where it will be applied.
\end{abstract}

Key words: Dental Veneers, Crown Lengthening, Composite Resins, Tooth Eruption.

\title{
1. Introducción
}

La erupción dentaria consta de dos fases bien diferenciadas: la erupción activa y la erupción pasiva. La primera es el movimiento que realiza el diente en sentido oclusal hasta contactar con su antagonista. Este proceso es seguido de la erupción pasiva, que consiste en la migración apical del complejo dentogingival hasta llegar a la línea amelocementaria. Así pues, según se produce el proceso de erupción pasiva, aumenta la longitud de la corona clínica. ${ }^{1}$ Este proceso se divide en cuatro fases; en la primera fase la unión dentogingival se encuentra en el esmalte, en la segunda la unión dentogingival se encuentra tanto en el esmalte como en el cemento, en la tercera la unión dentogingival se localiza en el cemento, extendiéndose coronalmente hasta la línea amelocementaria y en la cuarta fase la unión dentogingival se localiza en el cemento y la superficie radicular se encuentra expuesta por la migración apical de la unión dentogingival. ${ }^{1}$

Se considera que el margen gingival tiene que estar situado en condiciones normales a nivel o 1 milímetro coronal a la línea amelocementria en un individuo adulto sin pérdida de inserción. ${ }^{1}$ La erupción pasiva alterada (EPA) se desarrolla cuando el margen gingival se encuentra colocado incisal u oclusalmente sobre la corona anatómica y no migra apicalmente a una situación próxima al límite amelocementario, pudiendo ser la causa de la existencia de sonrisa gingival en gran número de pacientes. ${ }^{1}$ Para resolver casos de EPA, además de las carillas inyectadas, se puede realizar gingivectomía para obtener un resultado más estético, la utilización del electrobisturí hace el procedimiento menos invasivo, ofreciendo múltiples ventajas sobre la técnica convencional. ${ }^{2,3}$
Actualmente, una de las demandas más grandes en odontología son los tratamientos estéticos, los pacientes y odontólogos buscan que este tipo de tratamientos sean mínimamente invasivos o no invasivos. ${ }^{4}$ Existen muchos tratamientos en la actualidad para lograr una armonía estética, excelente función y preservación de los tejidos dentales. Así mismo, existen diferentes materiales para realizar carillas como son: resina compuesta, resina fluida, zirconia, porcelana, entre otros; el tipo de preparación será determinado de acuerdo al material a ser utilizado. ${ }^{4}$

Hoy en día se buscan técnicas menos invasivas, ya que algunos materiales requieren una preparación más extensa para su utilización. Por otro lado, algunos materiales pueden lograr su función con carillas muy delgadas que no necesitan preparación $^{5}$, además otro factor muy importante en las técnicas de carillas es la adhesión, según diversos autores la longevidad de la adhesión en esmalte es mucho mejor que en dentina, por lo que no se recomienda desgastar el tejido dentario en exceso. A su vez, se disminuye la posibilidad de que el paciente padezca algún tipo de sensibilidad postoperatoria. ${ }^{6}$ La técnica de carillas con resina inyectada es un proceso predecible tanto para el odontólogo como para el paciente ya que permite realizar una planeación con un encerado de diagnóstico y plasmar lo que se quiere obtener, por medio de un mockup. ${ }^{7,8}$ Debido a lo expuesto anteriormente, el objetivo del presente reporte de caso, es describir alternativa de rehabilitación estética y funcional de paciente joven que presentaba EPA por medio de un tratamiento mínimamente invasivo, utilizando la técnica de resinas inyectadas en el sector antero-superior. 


\section{Presentación del caso}

Paciente femenina de 17 años de edad, se presentó a la clínica de odontología de la Universidad Autónoma de Guadalajara referida por el departamento de ortodoncia. En la revisión clínica de la cavidad oral, la paciente presentaba diastemas en el sector antero-superior y el tratamiento de ortodoncia no era la primera opción ya que la paciente se encontraba en clase 1 molar y canina. En el motivo de la consulta la paciente refirió: "no me gustan los espacios que tengo entre mis dientes." Se procedió entonces a solicitar a la paciente un estudio periapical digital completo y se realizó un sondeo periodontal de los órganos dentarios $11,12,13,21,22$ y 23 . Se diagnosticó a la paciente con EPA, ya que la encía se encontraba por encima de la unión cemento esmalte (Figura 1).

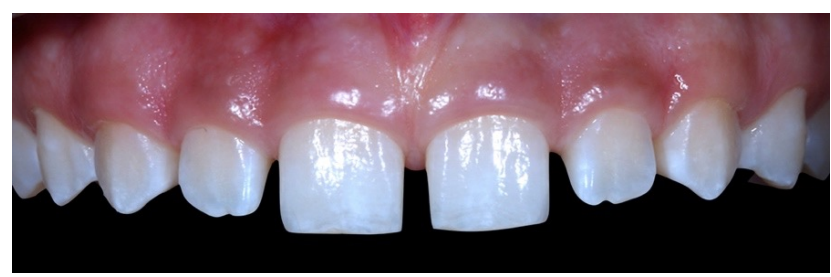

Fig. 1. Fotografía inicial que muestra diminución de coronas clínicas y presencia de diastemas.

Con el objetivo de brindarle el tratamiento ideal a la paciente se le explicó que se tenían qué realizar alargamientos de corona en el sector antero-superior, ya que presentaba un exceso de encía sobre sus dientes. Se anestesió (de forma infiltrativa) y se fue recorriendo el surco gingival presionando con la sonda periodontal hasta localizar la depresión existente a nivel de la unión amelocementaria. Estas mediciones permiten determinar la cantidad de encía que será necesario eliminar.

Para la realización de la gingivectomía se utilizó el mockup como guía quirúrgica y se marcó con una sonda periodontal donde se van a reposicionar los nuevos márgenes gingivales, se contorneó la encía con el electrobisturí y se fue tocando con movimientos de golpeteo o cepillado con intervalos de 1 a 1.5 segundos para disipar el calor. Este procedimiento se realizó en todo el sextante anterosuperior (Figura 2).

Se realizó la frenectomía, haciendo una incisión en forma romboide / balón de fútbol desde el frenillo hasta la encía adherida y el lado interno del labio, que se extiende hasta la profundidad del vestíbulo labial en la superficie labial hasta la punta incisiva de la papila en la superficie oclusal debido a que la papila penetra la unión del frenillo causando separación de fibras y liberación de tensión que estaba causando isquemia. ${ }^{5}$ La hemostasia fue óptima. Una vez terminado el procedimiento se citó a la paciente una semana después para revisión (Figura 2d).

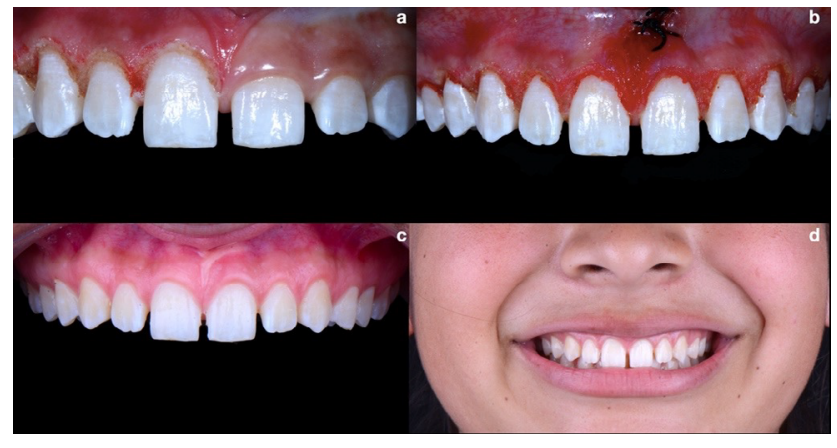

Fig. 2. (a) Alargamiento de corona realizado con electrobisturí, (b) Frenectomía realizada con electrobisturi, (c) Acompañamiento 3 semanas después de la intervención con la encía cicatrizada, (d) Fotografía de sonrisa después del procedimiento.

Después del período de cicatrización de los tejidos, fue discutido con la paciente el plan de tratamiento, donde se propuso que el tratamiento ideal serían las carillas de resina inyectada brindándole una rehabilitación estética y funcional, sin tener que realizar ningún tipo de desgaste dental y así se obtendría armonía en su sonrisa y presencia de guía anterior.

Luego de realizada la cirugía periodontal, se tomaron fotografías intraorales y extraorales, así como impresiones de polivinilsiloxano (3M ESPE) para elaborar modelos de diagnóstico, arco facial y registros interoclusales y se montaron los modelos en un articulador semiajustable (Panadent). Se realizó un diseño de sonrisa digital y se hizo el encerado de diagnóstico siguiendo parámetros estéticos. El mockup fue realizado con Bisacryl (Protemp $43 \mathrm{M}$ ESPE) en la boca del paciente para que pudiera observar el futuro resultado y opinar respecto a sus restauraciones, se verificó que la paciente tuviera desoclusiones posteriores en movimientos de lateralidad y protrusiva (Figura 3 ).

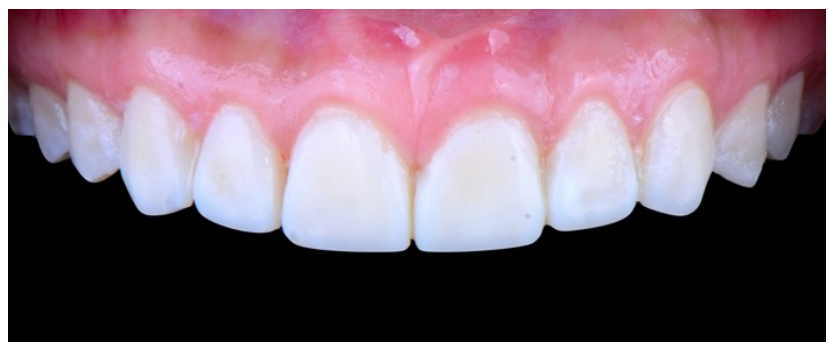

Fig. 3. Instalación del mock-up, para revisar parámetros estéticos $\mathrm{y}$ funcionales de las restauraciones 


\section{Proceso de laboratorio}

Para la realización de la matriz de acetato se desmontó el modelo superior y se realizó una llave de z-labor (silicona por condensación marca Zhermack) incluyendo aproximadamente la mitad del paladar y los primeros premolares para tener un tope vertical, después con la llave asentada se colocó un acetato semi-rígido calibre 40 y se llevó al vacuum, se retiró la llave de z-labor y se recortó el acetato. Se colocó el polivinixlsiloxano transparente (3M ESPE) en el acetato y se sumergió el modelo para posteriormente colocarlo en la olla de presión y se dejó 20 minutos a 20 libras de presión para evitar burbujas en la llave. Al sacarlo de la olla de presión se retiró el acetato y la matriz estaba lista. Posteriormente se perforó la llave en medio del borde incisal utilizando fresa de bola número 6, con pieza de mano de alta velocidad y se comprobó que permitiera el ingreso de la punta de una resina fluida (Figura 4).

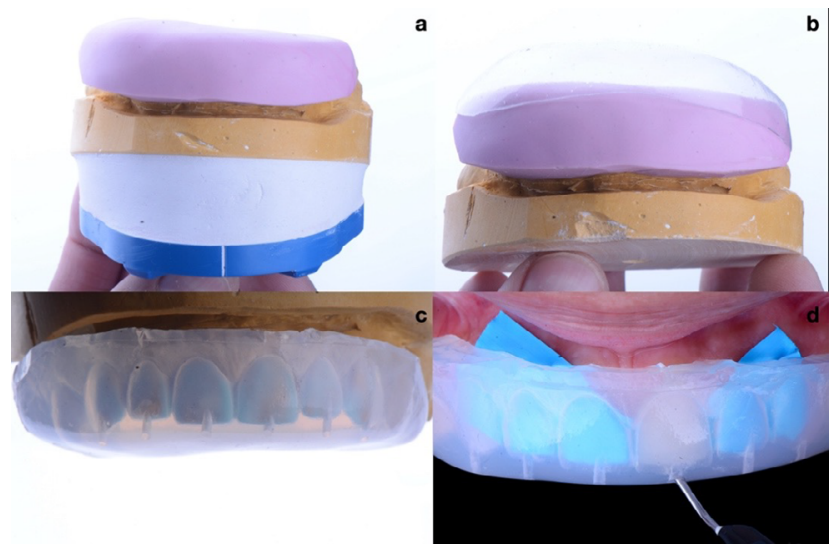

Fig. 4. (a) Matriz de Z labor, (b) Acetato calibre 40, (c) Matriz de polivinilsiloxano transparente, (d) infiltración de resina fluida.

\section{Proceso clínico}

Se llevó la llave de z-labor confeccionada a la boca del paciente para corroborar el excelente asentamiento, donde se verificó que incluyera por lo menos un diente adyacente a cada lado de los caninos y la mitad del paladar para tener un tope vertical de la matriz y no tener un exceso de presión al asentarla. Se pulieron los dientes con un cepillo de profilaxis y tierra pómez teniendo cuidado de no afectar la encía. Se colocó un optragate (Ivoclar Vivadent) para facilitar el proceso clínico y se puso cinta de teflón en los dientes adyacentes. Enseguida se realizó grabado ácido en el esmalte durante 20 segundos (ácido fosfórico Prodensa al 37\%) y se frotó con un ultrabrush para mejorar el grabado ácido. Se lavó él acido grabador con agua y aire durante 20 a 30 segundos y se secó con aire libre de aceite de la jeringa triple. Se colocó adhesivo en la superficie del esmalte (Scotchbond Universal Adhesivo 3M ESPE) y se frotó durante 20 segundos para que volatilizara el solvente, luego se secó con aire para después fotocurar durante 10 segundos según las indicaciones del fabricante.

Se posicionó la matriz e insertamos la punta de la resina fluida tratando de ubicarla en vestibular aproximadamente en el tercio medio coronal y se empezó a inyectar la resina fluida (Tetric N Flow Bleach L de Ivoclar) poco a poco hasta el tercio gingival y se retiró poco a poco la punta de la jeringa hasta ver que se inyectó completamente. Después se fotocuró durante 5 segundos y se retiró la matriz para quitar excedentes en la zona gingival con un bisturí número 12 (AMBIDERM) para seguir la anatomía gingival. Se retiraron los excesos en cada diente con hilo dental y se utilizó una lija interproximal en ambos lados. Después se fotocuró durante 20 segundos ( $3 \mathrm{~m}$ Elipar Deep Cure) por vestibular y 20 segundos por palatino, utilizando inhibidor de la capa de oxígeno (DeOx ULTRADENT) en toda la cara vestibular y palatino se procedió a fotocurar otros 20 segundos por cada cara. Se siguió el mismo procedimiento con los los 6 dientes anterosuperiores.

Al finalizar de inyectar se revisaron formas, tamaños y ángulos línea. Se removieron excesos cuidadosamente en la zona gingival para evitar una futura inflamación corroborando que no presentara ninguna interferencia que le impidiera una higiene dental adecuada. Enseguida se procedió con el proceso de pulido de las restauraciones en resina con un reductor de velocidad (friction grip NSK) e irrigación. El primer paso fue haciendo uso de discos SofLex (3M ESPE) de grano mayor a grano menor, luego se utilizó una borla de pelo de cabra, hules para resina y por último se colocó pasta diamantada en los dientes para darles el pulido final con una borla de pelo de borrego.

Se le dieron indicaciones a la paciente de no consumir en gran cantidad bebidas pigmentantes como café, té refresco de cola o vino tino y se le instruyó en cuanto a la técnica de cepillado, el uso de hilo dental y citas cada 3 o 4 meses para revisar la adaptación de las restauraciones, así como para poder realizar profilaxis y pulido para mantener la estabilidad de color (Figura 5).

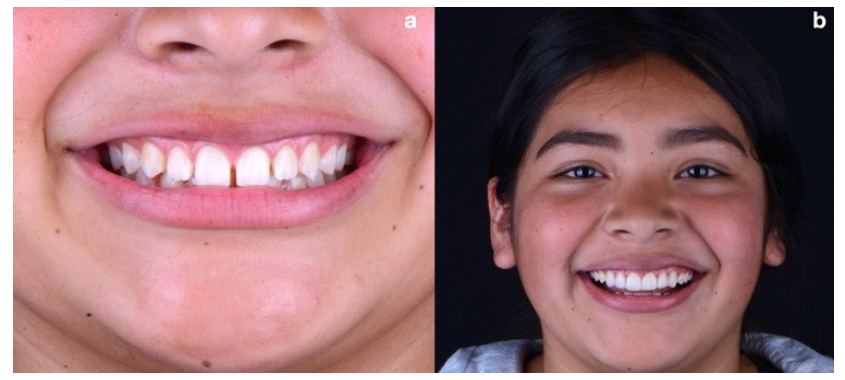

Fig. 5. Fotografía final (a) Antes y (b) Después, cumpliendo con parámetros estéticos y funcionales óptimos donde se muestra una sonrisa natural. 


\section{Discusión}

En la actualidad el exceso de encía al sonreir es uno de los motivos de consulta más comunes en la práctica clínica diaria; debido a la demanda estética de los pacientes. ${ }^{1} \mathrm{Se}$ tienen que tomar en cuenta las características extraorales como simetría facial, largo de la cara, anatomía del labio y línea de la sonrisa de los pacientes, así mismo es importante conocer las características intraorales como dimensión de los dientes, largo de las coronas anatómicas y de la corona clínica, contorno del tejido gingival, y topografía del hueso alveolar. $^{1}$

La electrocirugía no es una técnica ampliamente empleada en odontología, lo cual se puede deber a la necesidad de un láser y el poco conocimiento de esta técnica. En la actualidad ha evolucionado y los métodos clínicos se han refinado y modernizado. El procedimiento consiste en la aplicación de corriente de alta frecuencia, para incidir, coagular o disecar tejidos, permitiendo realizar incisiones estériles mientras se sellan los vasos sanguíneos y linfáticos del tejido. Esta técnica quirúrgica ofrece ventajas tales como un campo de trabajo más limpio por la hemostasia relativa que ofrece, mayor visibilidad, esterilización de la incisión, menos dolor postoperatorio, rapidez en el procedimiento y mayor comodidad para paciente y operador. ${ }^{2,3}$ Es una técnica predecible, fácil de manejar y cómoda tanto para el paciente como para el dentista debido al tiempo quirúrgico, poco sangrado y tiempo de cicatrización.,

En todos los tratamientos dentales se tendrá un manejo interdisciplinario del paciente para poder lograr un tratamiento final acertado y predecible a largo plazo. ${ }^{2}$ En los casos donde el paciente presenta EPA lo más indicado es hacer un alargamiento de corona. ${ }^{9}$ Así como tener un correcto diagnóstico y auxiliarse de la historia clínica completa, radiografías periapicales y sondear los órganos dentarios en los que se realizará el procedimiento restaurador. ${ }^{9} \mathrm{Al}$ ser una técnica poco invasiva, rápida y de fácil abordaje, fue la más indicada en este caso clínico. Los alargamientos de corona sin osteoplastia que se realizaron con electrobisturí tuvieron excelentes resultados, así mismo la cicatrización es mucho mas rápida y el postoperatorio es mucho mas cómodo para el paciente.

Siempre que sea posible, se deben situar los márgenes de las restauraciones supragingivales, ya que permite que el paciente tenga un adecuado acceso a higiene y pueda limpiar perfectamente la interfaz restauración-diente, y así la salud de los tejidos blandos circundantes será mas óptima a largo plazo sin presentar inflamación. ${ }^{9}$ En comparación con las técnicas convencionales que se utilizan para este tipo de casos como las carillas de porcelana, la técnica de resinas inyectadas es considerablemente más económica y requiere menos tiempo clínico. Además, las propiedades mecánicas, resistencia al desgaste, fuerza, pulibilidad, translucidez y otras características de los composites fluidos han mejorado considerablemente en los últimos años. La resina de composite fluida se considera más adecuada para su uso con un índice de silicona transparente debido a la facilidad de precisión y replicación del encerado de diagnóstico. ${ }^{10,11}$ Uno de los mayores retos es seleccionar el color adecuado para mimetizar la diferencia entre el esmalte y la resina.

La técnica empleada en la realización de este caso clínico se mostró eficaz debido a la corta edad de la paciente, con lo que se preservó el tejido dentario, evitando desgastes innecesarios, por lo tanto, puede utilizarse como un tratamiento únicamente aditivo, además de ser simple, de bajo costo y predecible a futuro debido a la prueba de mockup que se realiza previamente a las resinas inyectadas proponiendo así un plan de tratamiento con corto tiempo de resolución para el paciente. ${ }^{7,8}$ Una de las ventajas de esta técnica descrita por Douglas Terry es el uso de una matriz de polivinilsiloxano transparente que permite una visualización por completo al momento de la infiltración de la resina evitando así burbujas de aire, además también favorece durante el fotocurado permitiendo que la luz de la lámpara llegue perfectamente a todas las zonas donde se infiltra la resina. ${ }^{7,8}$

\section{Conflicto de intereses}

Los autores del presente estudio manifiestan que no existe ningún conflicto de intereses en relación al tema de estudio.

\section{Referencias}

1. Balda García Ignacio, Herrera Ureña José ignacio, Frías López María Cruz, Carasol Campillo Miguel. Erupción pasiva alterada: Implicaciones estéticas y alternativas terapéuticas. RCOE [Internet]. 2006 Dic [citado 2021 Abr 20] ; 11( 5-6 ): 563-571. Disponible en: http://scielo.isciii.es/scielo.php?scrip$\mathrm{t}=$ sci_arttext\&pid=S1138-123X2006000500005\&1$\mathrm{ng}=\mathrm{es}$.

2. Amaíz Flores Alejandro J.. La electrocirugía en la Odontología actual. Odontología Vital [Internet]. 2018 June [cited 2021 Apr 19]; ( 28 ): 91-101. Available from: http:/www.scielo.sa.cr/scielo.php?scrip$\mathrm{t}=$ sci_arttext\&pid=S1659-07752018000100091\&ln$\mathrm{g}=\mathrm{en}$. 
3. Vieyra BNL, Carrillo SC. Conceptos básicos de Recibido: 04 de junio de 2021 la electrocirugía en odontología restauradora. Rev ADM. 2001;58(6):206-219.

4. Gutiérrez-Romero F. Alargamiento de corona y gingivoplastia. Kiru. 2009; 6(1): 57-63 CROWN LENGTHENING. ALARGAMIENTO DE CORONA Y GINGIVOPLASTIA [Internet]. Edu.pe. 2009 [cited 2021 Apr 19]. Available from: https://www. usmp.edu.pe/odonto/servicio/2009/Kiru2009v6n1/ Kiru2009v6n1art9.pdf

5. Pandey S. Laser Frenectomy of Papilla Penetrating Frenum - A Case Report. Act Scie Dental. 2019;3(11):22-24.

6. Christensen GJ. Bonding to dentin and enamel where does it stand in 2005? J Am Dent Assoc. 2005;136(9):1299-1302.

7. Terry DA, Powers J. Using injectable resin composite: part one. International Dentistry-African edition. Vol. 5 NO.1: 52-62 Terry D, Powers J. Using injectable resin composite: part one [Internet]. Moderndentistrymedia.com. [cited 2021 Apr 19]. Available from: http://www.moderndentistrymedia.com/ jan_feb2015/terry_part-one.pdf

8. Terry DA. Developing a functional composite resin provisional. Am J Esthet Dent 2012;2:56-66 Terry DA. Developing a functional composite resin provisional [Internet]. Dentalinstitute.com. [cited 2021 Apr 19]. Available from: https://dentalinstitute.com/ wp-content/uploads/2019/12/ajed_2_1_Terry_6.pdf

9. Herrera-Tituana SX. Comparación entre carillas de resina y carillas de porcelana (tesis de licenciatura en Protesis Dental Fija) Ciudad de Guayaquil. Universidad de Guayaquil. 2009.

10. Geštakovski D. The injectable composite resin technique: minimally invasive reconstruction of esthetics and function. Clinical case report with 2-year follow-up. Quintessence Int. 2019;50(9):712-719.

11. Magne P, Douglas WH. Additive contour of porcelain veneers: a key element in enamel preservation, adhesion, and esthetics for aging dentition. J Adhes Dent. 1999 Spring;1(1):81-92. 